\title{
Uniprojective Features for Gait Recognition
}

\author{
Daoliang Tan, Kaiqi Huang, Shiqi Yu, and Tieniu Tan \\ Center for Biometrics and Security Research, \\ National Laboratory of Pattern Recognition, Institute of Automation, \\ Chinese Academy of Sciences, Beijing, P.R. China, 100080 \\ $\{$ dltan, kqhuang, sqyu, tnt $\} @$ nlpr.ia.ac.cn \\ http://www.cbsr.ia.ac.cn
}

\begin{abstract}
Recent studies have shown that shape cues should dominate gait recognition. This motivates us to perform gait recognition through shape features in 2D human silhouettes. In this paper, we propose six simple projective features to describe human gait and compare eight kinds of projective features to figure out which projective directions are important to walker recognition. First, we normalize each original human silhouette into a square form. Inspired by the pure horizontal and vertical projections used in the frieze gait patterns, we explore the positive and negative diagonal projections with or without normalizing silhouette projections and obtain six new uniprojective features to characterize walking gait. Then this paper applies principal component analysis (PCA) to reduce the dimension of raw gait features. Finally, we recognize unknown gait sequences using the Mahalanobis-distance-based nearest neighbor rule. Experimental results show that the horizontal and diagonal projections have more discriminative clues for the side-view gait recognition and that the projective normalization generally can improve the robustness of projective features against the noise in human silhouettes.
\end{abstract}

Keywords: Gait recognition, projective features, PCA.

\section{Introduction}

In a broad sense, gait means the moving style on bodies and involves dynamic and static features. Cutting and Kozlowski 3 found out from their cognitive experiments that gait can be used to recognize the identity of walking people. It is this psychological finding that inspires many computer vision researchers to pursue gait-based walker recognition.

There has thus far been a body of work in the gait recognition field. For example, Cunado et al. 2] described the motion of human legs using a model of two connected pendulums and distinguished walkers using the Fourier transform coefficients of the thigh movement in the low frequency band. Later, Urtasun and Fua 9 built on a 3D model to characterize the physical structure of human bodies and recognized unknown gait patterns with the help of a vector of 84 angles in the 3D model. By contrast, Liu et al. 5] used projective frieze patterns to represent gait signatures. In addition, Kale et al. 4 encoded human silhouette 
gait into a group of distances in the horizontal direction. Furthermore, Wang et al. 11] employed contour-to-centroid distances to depict human figures (shapes). In particular, Veeraraghavan et al. [10] revealed that shape clues dominate gait recognition in comparison with dynamic features. On the other hand, it seems that dynamic features are susceptible to behavioral changes from the long-term point of view.

The success of shape features in gait recognition, together with the simplicity and robustness of projective frieze patterns [5], motivates us to recognize walking people through projective features in 2D human silhouettes. In this paper, we propose six projection-based features to describe human gait and compare them with frieze features to figure out which of the projective directions are important to walker recognition.

The rest of this paper is organized as follows. Section 2 introduces our method. Then, In Section 3, we examine the method on three well-known gait databases. Finally, Section 4 concludes this paper.

\section{Method}

Since current gait databases 178 provide segmented silhouettes apart from raw video sequences, this paper will focus on gait representation, dimension reduction, and identity recognition. First of all, we normalize each human silhouette image into a square form of $32 \times 32$ shown in Fig. 1. Then we project the normalized silhouette in four directions: horizontal $(\rightarrow)$, vertical $(\downarrow)$, positive diagonal $(\searrow)$, and negative diagonal $(\nearrow)$; we use the vector of projective values in each projection direction to represent human gait. Furthermore, we apply principal component analysis (PCA) to reduce the dimension of the original feature vector. Finally, we exploit the simple nearest neighbor rule to classify the identity of unknown gait.

\subsection{Gait Representation}

We can obtain eight projective representations, two of which are the same as the frieze features [5], with or without projection normalization. More specifically, given a normalized silhouette, we can evaluate for each projective direction a maximum indicating the largest number of foreground pixels in that direction and choose whether to normalize the projection by the maximum or not. In addition, this paper uses the symbols $\mathrm{H}, \mathrm{V}, \mathrm{PD}$, and ND to denote the $\rightarrow$, $\downarrow$, \, and $\nearrow$ projections without normalization, respectively; the normalized projections are expressed as the star versions: $\mathrm{H}^{*}, \mathrm{~V}^{*}, \mathrm{PD}^{*}$, and $\mathrm{ND}^{*}$. Figure 1 illustrates the eight projective features in the form of curves.

\subsection{Dimension Reduction}

The aim of this step is to reduce the redundancy in the projective feature vector $x \in \mathcal{R}^{n}$ for the benefit of lower computational complexity. Moreover, since the distance measure used involves the second-order covariance matrix, it naturally 


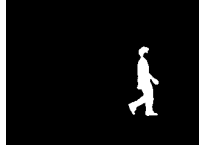

(a)

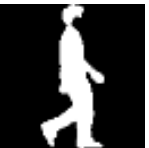

(b)

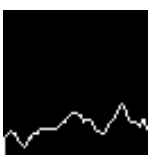

(c)

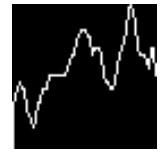

(g)

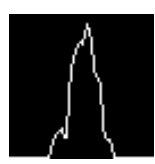

(d)

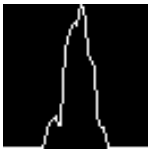

(h)

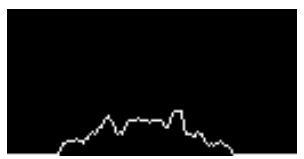

(e)

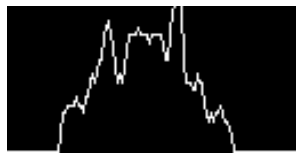

(i)

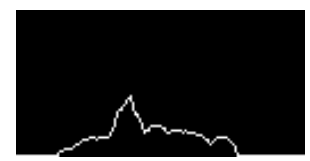

(f)

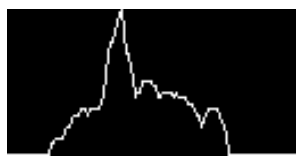

(j)

Fig. 1. Illustration of eight projective gait features. (a) Initial silhouette. (b) Normalized silhouette. (c) H. (d) V. (e) PD. (f) ND. (g) H*. (h) V*. (i) PD*. (j) ND*.

seems more favorable to perform this operation in return for the lower-order covariance matrix.

More precisely, we take advantage of PCA to accomplish this aim, since PCA has an analytical form and small computational burden. This paper presents the derivation of the PCA-transformation matrix $W \in \mathcal{R}^{n \times d}$ from the variance maximization criterion. Assume that $W$ can be written as $W=\left[w_{1} \ldots w_{d}\right]$ and we have known $k-1$ columns $\left(w_{1}, \ldots, w_{k-1}, k \leq d\right)$. The next is to determine the remaining $d-k+1$ columns $\left(w_{k}, \ldots, w_{d}\right)$. We can concisely formulate this problem as (1):

$$
\max _{w_{k} \in \mathcal{R}^{n}} \operatorname{var}\left(w_{k}^{T} x\right) \quad \text { s.t. } w_{k}^{T} w_{k}=1, w_{k}^{T} w_{l}=0 \quad(l=1, \cdots, k-1)
$$

where $k$ ranges from 2 to $d$. Furthermore, we can reduce the constrained optimization problem (11) to the unconstrained one (2) by introducing Lagrange multipliers:

$$
\min f\left(w_{k}, \lambda_{1}, \ldots, \lambda_{k}\right)=-w_{k}^{T} C_{x} w_{k}+\lambda_{k}\left(w_{k}^{T} w_{k}-1\right)+\sum_{l=1}^{k-1} \lambda_{l} w_{k}^{T} w_{l}
$$

where $C_{x}$ is the covariance matrix of the vector $x$ and $\lambda_{l}(l=1,2, \ldots, k)$ is the Lagrange multiplier. Differentiating $f\left(w_{k}, \lambda_{1}, \ldots, \lambda_{k}\right)$ with respect to $w_{k}$ and $\lambda_{l}(l=1,2, \ldots, k)$ to be zero yields that the optimal $w_{k}$ and $\lambda_{k}$ compose an eigenpair of $C_{x}$, with $\lambda_{k}$ being the $k$-th largest eigenvalue of $C_{x}$. Thus the mapping $W$ belongs to the column-orthogonal matrices (i.e., $W^{T} W=I$ ) and has as its columns $w_{1}, w_{2}, \ldots, w_{d}$. In this paper, we make $d$ be the minimum number of components of $x$ needed to explain at least a fraction $95 \%$ of the 
total variation of $x$ [6]. Finally, we can project $x$ onto a subspace spanned by the columns of $W$ using the mapping $y=W^{T} x$.

\subsection{Identity Recognition}

For a gait sequence of $N$ frames, this paper coarsely simplifies its description using the average feature vector $\bar{y}$ shown in Eq. (3):

$$
\bar{y}=\frac{1}{N} \sum_{t=1}^{N} W^{T} x_{t}
$$

where $x_{t}$ is an original feature vector in the sequence at time $t$. Moreover, we employ the Mahalanobis distance to evaluate the similarity measure, since the Mahalanobis measure generally can produce a better and more robust performance in our extensive experiments, compared with the $L_{1}, L_{2}$, and $L_{\infty}$ metric. Finally, this paper classifies the identity of unknown gait based on the nearest neighbor rule.

\section{$3 \quad$ Experiments}

In order to figure out which projective features or directions are important to gait recognition, we compare the performance of the eight projective features on three important gait databases: CMU Mobo Database [1], USF-NIST Gait Database [7], and CASIA Infrared Night Gait Dataset [8]. In addition, cumulative match scores (CMS) are used to quantitatively assess the recognition performance. Here the CMS value $\alpha$ corresponding to rank $r$ indicates a fraction $100 \cdot \alpha \%$ of probes whose top $r$ matches must include the real identity matches. The following will give more experimental details.

\subsection{CMU Mobo Gait Database}

This database consists of gait sequences - captured in an indoor scenario - from a small number of 25 subjects and records four walking conditions: slow walking, fast walking, slow walking at a certain slope, and slow walking with a ball. Figure 2 displays unnormalized sample silhouette images in the Mobo database. We carry out two types of experiments based on the side-view gait sequences in this database: within-condition tests, where the training and testing data has the same walking attribute, and across-condition tests, where the training and testing data has different walking attributes. More specifically, in the withincondition cases, this paper employs the first half part of each subject's sequence to be the training data and the remaining half sequence to be the testing data, whereas we exploit the whole sequences of each subject for training or testing in the across-condition cases [4.

Furthermore, Table 1 presents five experiments proposed by Kale et al. 4] on this database, and Table 2 compares the rank 1 performance (correct recognition 


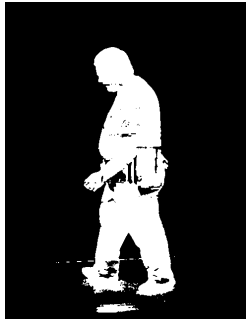

(a)

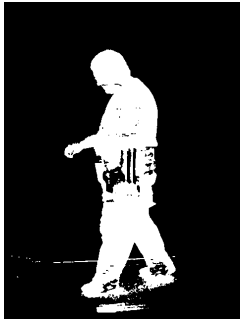

(b)

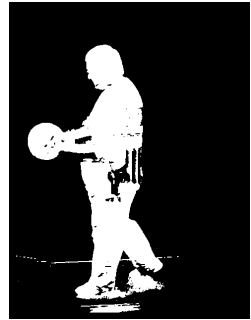

(c)

Fig. 2. Sample silhouette images in the CMU Mobo Database. (a) Fast walking. (b) Slow walking. (c) Slow walking with a ball.

Table 1. Five Experiments on the CMU Mobo Gait Database

\begin{tabular}{|c|c|c|c|}
\hline Exp. & Gallery & Probe & Type \\
\hline A & Slow & Slow & Within-condition \\
\hline B & Fast & Fast & Within-condition \\
\hline C & Ball & Ball & Within-condition \\
\hline D & Slow & Fast & Across-condition \\
\hline E & Fast & Slow & Across-condition \\
\hline
\end{tabular}

rates) of the eight projective features and the baseline method 4. It can be seen from Table 2 that the $\mathrm{V}$ and $\mathrm{V}^{*}$ representations are inferior to other six projective features but comparable to the baseline method in terms of the recognition accuracy, though our method depends not on an HMM to simulate the temporal relations in the gait movement. Moreover, Figure 3 depicts the CMS curves of the eight projective gait features in the five experiments and illustrates the fast convergence of the CMS values toward the unity. In addition, the recognition in the across-condition case poses a much greater challenge than in the withincondition case owing to the variation in walking attributes. Finally, both Table 2 and Fig. 3 show that the horizontal and positive diagonal directions have more important discriminating clues.

\subsection{USF-NIST Gait Database}

After considering the issues about storage capacity and computational time, we make direct use of the precomputed silhouettes for the May-2001-No-Briefcase data of a moderate size in the USF-NIST Gait Database [7. Figure 4 displays four noisy sample walker silhouettes in this database. The gait collection used in this paper includes 74 individuals and has such factors as view (left and right), footwear (two types of shoes), and ground surface (concrete and grass). Sarkar et al. 7] designed seven challenging recognition experiments shown in Table 3 for this data collection so as to benchmark the assessment of gait recognition algorithms. 
Table 2. A Comparison of the Rank 1 Performance of nine gait features on the Mobo Gait Database

\begin{tabular}{|c|c|c|c|c|c|}
\hline & $\mathrm{A}$ & $\mathrm{B}$ & $\mathrm{C}$ & $\mathrm{D}$ & $\mathrm{E}$ \\
\hline HMM [ & $72 \%$ & $68 \%$ & $92 \%$ & $32 \%$ & $56 \%$ \\
\hline $\mathrm{H}$ & $100 \%$ & $100 \%$ & $96 \%$ & $96 \%$ & $92 \%$ \\
\hline $\mathrm{V}$ & $100 \%$ & $96 \%$ & $92 \%$ & $44 \%$ & $60 \%$ \\
\hline $\mathrm{PD}$ & $100 \%$ & $96 \%$ & $100 \%$ & $92 \%$ & $72 \%$ \\
\hline $\mathrm{ND}$ & $100 \%$ & $100 \%$ & $96 \%$ & $72 \%$ & $60 \%$ \\
\hline $\mathrm{H}^{*}$ & $100 \%$ & $100 \%$ & $96 \%$ & $92 \%$ & $92 \%$ \\
\hline $\mathrm{V}^{*}$ & $92 \%$ & $88 \%$ & $88 \%$ & $32 \%$ & $48 \%$ \\
\hline $\mathrm{PD}^{*}$ & $100 \%$ & $96 \%$ & $96 \%$ & $80 \%$ & $80 \%$ \\
\hline $\mathrm{ND}^{*}$ & $100 \%$ & $100 \%$ & $96 \%$ & $72 \%$ & $60 \%$ \\
\hline
\end{tabular}

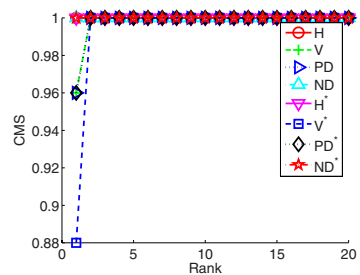

(a) Exp. A

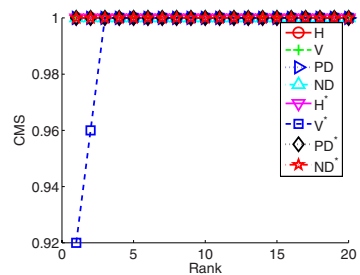

(b) Exp. B

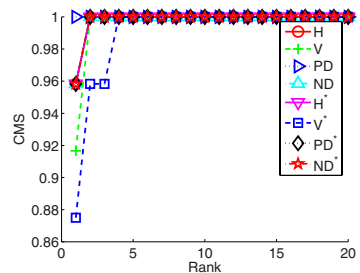

(c) Exp. C

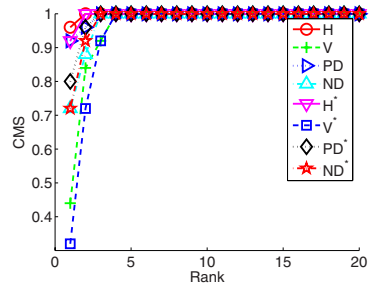

(d) Exp. D

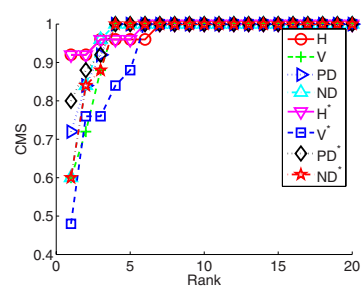

(e) Exp. E

Fig. 3. CMS curves on the CMU Mobo Database

Figure 5 compares the CMS performance of the eight projective features and the baseline algorithm [7] denoted by BL in the legends. We can draw four points from Fig. 5. First, the projective features except for $\mathrm{V}$ and $\mathrm{V}^{*}$ have the better recognition rates (CMS at rank 1) than BL in Exps. A, B, and C. Second, the recognition rate of $\mathrm{BL}$ is superior to the eight features in Exps. D, E, and $\mathrm{F}$ and to the $\rightarrow$ and $\downarrow$ projective features in Exp. G. Third, the normalized features are more robust against the segmenting noise (due to surface changes) than the unnormalized features in Exps. D, E, F, and G. Last but not least, the eight gait features exhibit more rapid CMS rises than the BL method.

As far as recognition results from this database are concerned, it appears that view variation has the weakest impact and ground surface types have 


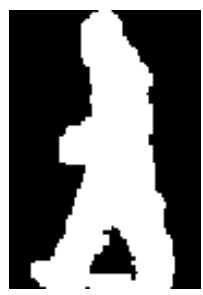

(a)

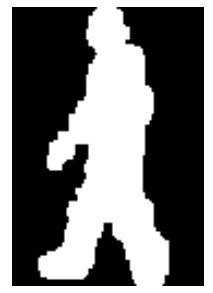

(b)

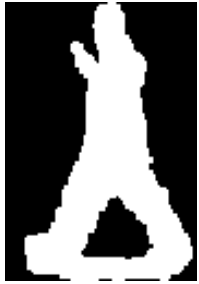

(c)

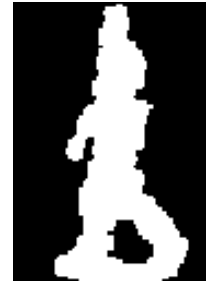

(d)

Fig. 4. Sample silhouette images from the USF-NIST Gait Database. (a) Left view on the grass surface. (b) Right view on the grass surface. (c) Left view on the concrete surface. (d) Right view on the concrete surface.

Table 3. Seven Experiments on the USF-NIST Gait Database

\begin{tabular}{|c|c|c|}
\hline Exp. & Probe & Difference \\
\hline A & $(\mathrm{G}, \mathrm{A}, \mathrm{L})$ & View \\
\hline B & $(\mathrm{G}, \mathrm{B}, \mathrm{R})$ & Shoe \\
\hline C & $(\mathrm{G}, \mathrm{B}, \mathrm{L})$ & Shoe,, View \\
\hline D & $(\mathrm{C}, \mathrm{A}, \mathrm{R})$ & Surface \\
\hline E & $(\mathrm{C}, \mathrm{B}, \mathrm{R})$ & Surface, Shoe \\
\hline F & $(\mathrm{C}, \mathrm{A}, \mathrm{L})$ & Surface, View \\
\hline G & $(\mathrm{C}, \mathrm{B}, \mathrm{L})$ & Surface, Shoe, View \\
\hline
\end{tabular}

the strongest impact on appearance-based gait recognition algorithms. The real reason for the vital effect of ground surface is, in our opinion, that surface type changes can induce great silhouette variation originating from inconsistent segmentation errors. It is this drastic silhouette changes that take the responsibility for the decline in the precision of recognition results.

\subsection{CASIA Infrared Night Gait Dataset}

This dataset comprises the night gait sequences of 153 subjects and takes into account four classes of walking patterns: normal walking, slow walking, fast walking, and normal walking with a bag. Each subject has ten sequences of his or her own gait: four sequences for the normal walking case and two sequences for each of the other walking cases. Figure 6 shows some unnormalized binary profile images of one person within this dataset. Furthermore, Table 4 lists four experiments on this gait dataset: we use the set of the first two normal-walking sequences of each person as the training data and the remaining sequences as the testing data. More precisely, Exp. A is concerned with the recognition performance of different projective features in the normal walking case. The purpose of Exps. $\mathrm{B}$ and $\mathrm{C}$ is to examine the effect of walking paces on recognition accuracy. Finally, the focus of Exp. D is on the degree to which appearance variation affects the gait-recognizing performance. 


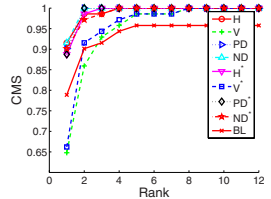

(a) Exp. A

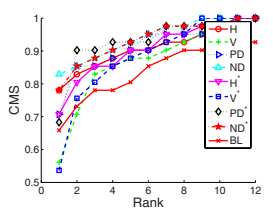

(b) Exp. B

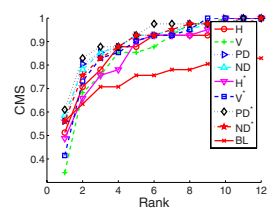

(c) Exp. C

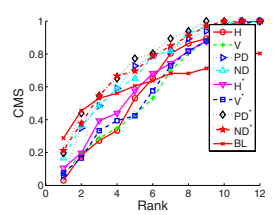

(d) Exp. D

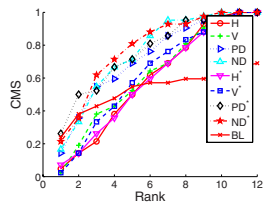

(e) Exp. E

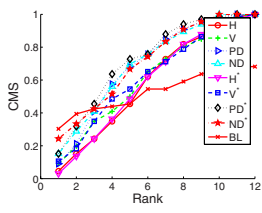

(f) Exp. F

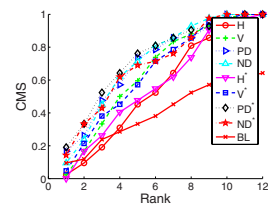

(g) Exp. G

Fig. 5. CMS curves on the USF-NIST Gait Database

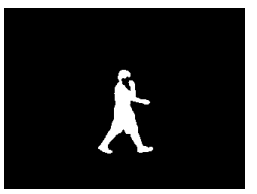

(a)

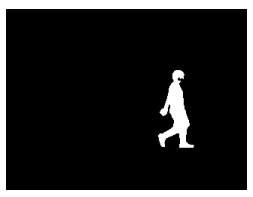

(b)

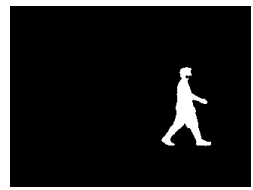

(c)

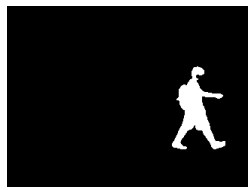

(d)

Fig. 6. Sample silhouette images in the CASIA Night Gait Dataset. (a) Normal walking. (b) Fast walking. (c) Slow walking. (d) Normal walking with a bag.

Figure 7 delineates the CMS values in response to the rank ranging from 1 to 20 . We can notice from Fig. 7 that the normalization step in projecting human silhouettes generally can improve recognition performance. Additionally, the $\rightarrow$, \, and $\nearrow$ projections can produce more useful features than the $\downarrow$ one. Furthermore, the changes in walking attributes more or less affect the recognition accuracy due to the chain-linked appearance variation. In general, the projective gait features other than the $\downarrow$ one can yield acceptable recognition performance, in view of the large number of subjects in this gait dataset. In particular, the $\rightarrow$ features can give the best performance in the four experiments on this dataset.

\subsection{Discussions}

As far as the current experimental results are concerned, it seems that the $\rightarrow$, $\searrow$, and $\nearrow$ features have more discriminative clues for the side-view gait, but this does not mean that the $\downarrow$ features play a less important role in gait recognition. In fact, we found out in other experiments (omitted here) that the $\downarrow$ projections can bring good recognition performance for the frontal-view gait. In a word, there does not exist one projective feature or direction winning all the contests.

In addition, the $30^{\circ}$ view difference [7 may be not large enough to cause great changes in appearance features and further to deteriorate recognition accuracy, according to the recent multiview gait recognition experiments by Yu et al. [12]. 
Table 4. Four experiments on the CASIA Infrared Night Gait Dataset

\begin{tabular}{|c|c|c|c|c|}
\hline Exp. & Gallery & Probe & \#Gallery Seq. & \#Probe Seq. \\
\hline A & Normal & Normal & 306 & 306 \\
\hline B & Normal & Fast & 306 & 306 \\
\hline C & Normal & Slow & 306 & 306 \\
\hline D & Normal & Bag & 306 & 306 \\
\hline
\end{tabular}

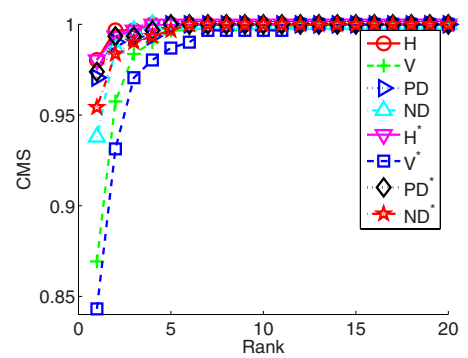

(a) Exp. A

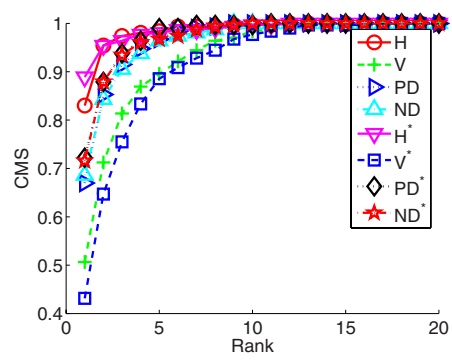

(c) Exp. C

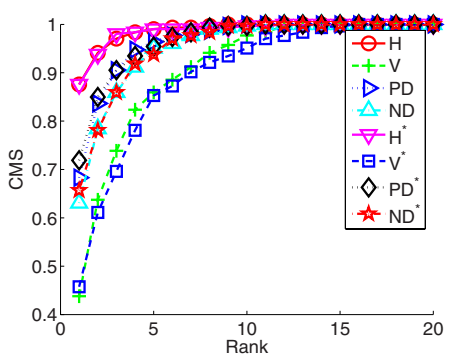

(b) Exp. B

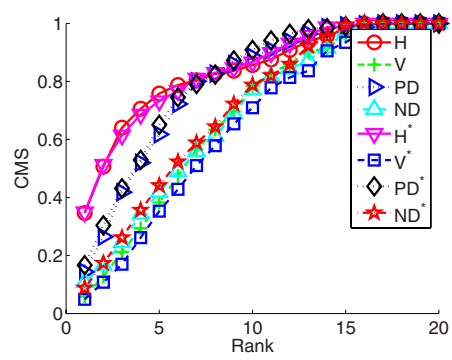

(d) Exp. D

Fig. 7. CMS curves on the CASIA Night Gait Dataset

It is thoughtless to conclude just based on current results that surface types are more crucial than view to appearance-based gait recognition. The general point is, in our eyes, that care should be taken of those factors which have an important effect on silhouette generation.

\section{Conclusions}

This paper has discussed the performance of eight uniprojetive shape features for gait recognition. Experimental results show that the horizontal and diagonal projections play a more critical role in the recognition performance than the vertical projection for the side-view walking gait recognition. In addition, the projection normalization can further enhance the robustness of the projective features against the noise in human silhouettes in terms of the correct recognition accuracy. Our contribution lies in the five promising uniprojective features for 
gait recognition. A natural idea will be to combine these uniprojective features and make full use of their respective advantages. We will look into this issue in our future work.

Acknowledgments. This work is funded by the National Natural Science Foundation of China (Grant No. 60605014, 60332010, and 60335010), the National Basic Research Program of China (Grant No. 2004CB318110), China International Science and Technology Cooperation (Grant No. 2004DFA06900), and the CASIA Innovation Fund for Young Scientists.

\section{References}

1. Collins, R., Gross, R., Shi, J.: Silhouette-based human identification from body shape and gait. In: Proc. Automatic Face and Gesture Recognition, pp. 366-371 (2002)

2. Cunado, D., Nixon, M., Carter, J.: Automatic extraction and description of human gait model for recognition purposes. CVIU 90(1), 1-41 (2003)

3. Cutting, J.E., Kozlowski, L.T.: Recognizing friends by their walk: Gait perception without familarity cues. Bulletin of the Psychonomic Society 9(5), 353-356 (1977)

4. Kale, A., Sundaresan, A., Rajagopalan, A., Cuntoor, N., RoyChowdhury, A., Krueger, V.: Identification of humans using gait. IEEE Trans. Image Processing 13(9), 1163-1173 (2004)

5. Liu, Y., Collins, R., Tsin, Y.: Gait sequence analysis using frieze patterns. In: Heyden, A., Sparr, G., Nielsen, M., Johansen, P. (eds.) ECCV 2002. LNCS, vol. 2351, Springer, Heidelberg (2002)

6. Mardia, K.V., Kent, J.T., Bibby, J.M.: Multivariate analysis, pp. 242-243. Academic Press, London (1979)

7. Sarkar, S., Philips, P., Liu, Z., Vega, I., Grother, P., Bowyer, K.: The human gait challenge problem: data sets, performance and analysis. PAMI 27(2), 162-177 (2005)

8. Tan, D., Huang, K., Yu, S., Tan, T.: Efficient night gait recognition based on template matching. In: Proc. ICPR, pp. 1000-1003 (2006)

9. Urtasun, R., Fua, P.: 3d tracking for gait characterization and recognition. In: Proc. Automatic Face and Gesture Recognition, pp. 17-22 (2004)

10. Veeraraghavan, A., Roy-Chowdhury, A., Chellappa, R.: Matching shape sequences in video with applications in human movement analysis. PAMI 27(12), 1896-1909 (2005)

11. Wang, L., Tan, T., Hu, W., Ning, H.: Silhouette analysis-based gait recognition for human identification. PAMI 25(12), 1505-1518 (2003)

12. Yu, S., Tan, D., Tan, T.: Modelling the effect of view angle variation on appearancebased gait recognition. In: Narayanan, P.J., Nayar, S.K., Shum, H.-Y. (eds.) ACCV 2006. LNCS, vol. 3851, pp. 807-816. Springer, Heidelberg (2006) 